\title{
A Fourth-Order Accurate Numerical Boundary Scheme for the Planar Dielectric Interface: a 2-D TM Case
}

\author{
Kyu-Pyung Hwang
}

\begin{abstract}
Preserving high-order accuracy in high-order FDTD solutions across dielectric interfaces is very important for practical time-domain electromagnetic simulations. This paper presents a fourth-order accurate numerical boundary scheme for the planar dielectric interface to be used in the fourth-order FDTD method proposed earlier by the author. The interface scheme for the two-dimensional (2-D) transverse magnetic (TM) polarization case is derived and validated by monitoring the $L_{2}$ norm errors in the numerical solutions of a partially-filled cavity demonstrating its fourth-order convergence and long-time numerical stability in the presence of the planar dielectric interface.

Key words : Finite-Difference Time-Domain (FDTD) Methods, Convergence of Numerical Methods, High-Order Method, Numerical Stability.
\end{abstract}

\section{Introduction}

Demand for high-order finite-difference time-domain (FDTD) methods often arises from electromagnetic problems that involve large scale full-wave solutions of time-dependent Maxwell's equations. At present, various forms of high-order FDTD methods have been proposed to deliver time-domain electromagnetic solutions with high-order accuracy [1 9]. In the presence of heterogeneous media within the computational domain, maintaining high-order accuracy across material interfaces is crucial for justifying the practical applications of high- order FDTD schemes. To address this problem, significant contributions have been made to assure high-order convergence globally in high-order differential stencils [3 9]. One-sided difference approximations and extrapolations can be used to implement numerical boundary conditions across dielectric interfaces for uniform fourthorder convergence [3 5]. The author has proposed a semi-implicit boundary scheme to realize fourth- order accuracy across dielectric interfaces, which results in local modifications of the fourth-order FDTD equations originally derived in a homogeneous medium [8, 9].

The boundary scheme for the material interface presented in the previous work [8,9] is only applicable to a two-dimensional (2-D) transverse electric (TE) polarization case. In the present paper, the boundary scheme is extended to the 2-D transverse magnetic (TM) case. A set of update equations for the electric field nodes along the planar interface is derived using high-order numerical integrations for proper handling of the field discontinuities. This interface update step is followed by a stabilizer step to guarantee long-time stability of the high-order FDTD solutions. Numerical tests in a 2-D partially-filled cavity are conducted for validation of the proposed boundary treatment.

\section{II . FDTD Equations}

Maxwell's equations for the 2-D TM case can be written in an isotropic, homogeneous, lossless medium as

$$
\frac{\partial \mathbf{U}}{\partial t}=L(\mathbf{U})
$$

where

$$
\mathbf{U}=\left[\begin{array}{l}
H_{x} \\
H_{y} \\
E_{z}
\end{array}\right], \quad L(\mathbf{U})=\left[\begin{array}{l}
-\frac{1}{\mu} \frac{\partial E_{z}}{\partial y} \\
\frac{1}{\mu} \frac{\partial E_{z}}{\partial x} \\
-\frac{1}{\varepsilon} \frac{\partial H_{x}}{\partial y}+\frac{1}{\varepsilon} \frac{\partial H_{y}}{\partial x}
\end{array}\right] .
$$

Since this is the complementary case of the 2-D TE polarization considered previously $[8,9]$, the fourthorder discretizations can be done in a similar manner to derive update equations for electric and magnetic field nodes in free space or in an inner domain away from boundaries, as well as in the vicinity of the dielectric

Manuscript received November 15, 2010 ; revised February 21, 2011. (ID No. 20101115-034J)

Samsung Electro-Mechanics Co., Ltd., Suwon, Korea.

Corresponding Author : Kyu-Pyung Hwang (e-mail : kphwang@ieee.org) 


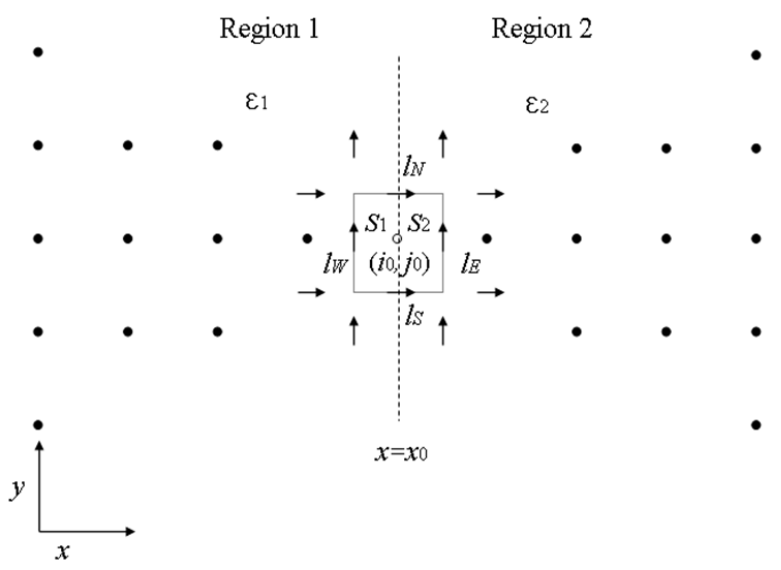

Fig. 1. Numerical stencil for the electric field, $\widehat{E}_{z i_{0}, j_{0}}^{n+1}$ denoted by the unfilled circle at the planar dielectric interface. Filled circles indicate electric field nodes with known $n+1$ time level information. Arrows in the $x$ and $y$ directions represent magnetic field nodes with known $n+1 / 2$ time level information.

and metallic boundaries. The fourth-order staggered backward differentiation method [10] and the fourth-order staggered central difference are used for temporal and spatial approximations, respectively. For electric and magnetic nodes near metallic or dielectric boundaries, the update equations are locally modified by a one-sided staggered difference approximation in space in order to maintain the fourth-order accuracy [8,9]. The results of analysis with regard to numerical dispersion and stability reported [9] previously remain valid for the 2-D TM formulation.

A major challenge in implementing the fourth-order FDTD method in the 2-D TM case lies in the interface scheme for the tangential electric field. To derive the update equation for the tangential electric field component, $E_{z}$ at the interface node $\left(x_{0}, y_{0}\right)=\left(i_{0} \Delta x, j_{0} \Delta y\right)$ as illustrated in Fig. 1, we start from the integral form of Ampere's law

$$
\frac{\partial}{\partial t} \iint_{S} \mathbf{D} \bullet \mathbf{d} \mathbf{s}=\oint_{C} \mathbf{H} \bullet \mathbf{d} \mathbf{l}
$$

In the finite-volume cell for $E_{z i_{0}, j_{0}}^{n+1}$, (3) can be rewritten as

$$
\begin{aligned}
& \frac{\partial}{\partial t}\left[\varepsilon_{1} \iint_{S_{1}} E_{z}(x, y) d x d y+\varepsilon_{2} \iint_{S_{2}} E_{z}(x, y) d x d y\right] . \\
& =\int_{l_{S}} H_{x} d x-\int_{l_{N}} H_{x} d x-\int_{l_{W}} H_{y} d y+\int_{l_{E}} H_{y} d y
\end{aligned}
$$

Unlike the case for the previous 2-D TE formulation, we have surface integrals that need to be evaluated on rectangular domains. Numerical evaluations of surface and line integrals in (4) need to have high-order accuracy to accomplish the overall fourth-order accuracy in space and time. For line integrals along the four segments around the rectangular cell, a three- point numerical integration technique is used leading to

$$
\begin{aligned}
& \int_{l_{S}} H_{x} d x-\int_{l_{N}} H_{x} d x-\int_{l_{W}} H_{y} d y+\int_{l_{E}} H_{y} d y \\
& =\Psi_{x} H_{x_{i_{0}}, j_{0}-1 / 2} \Delta x-\Psi_{x} H_{x_{i_{0}}, j_{0}+1 / 2} \Delta x- \\
& \Psi_{y} H_{y_{i_{0}-1 / 2, j_{0}}} \Delta y+\Psi_{y} H_{y_{i_{0}+1 / 2, j_{0}}} \Delta y \\
& +O\left((\Delta x)^{6}+(\Delta y)^{6}\right)
\end{aligned}
$$

where

$$
\begin{aligned}
& \Psi_{x} f_{i, j}=\frac{1}{24}\left(f_{i-1, j}+22 f_{i, j}+f_{i+1, j}\right), \\
& \Psi_{y} f_{i, j}=\frac{1}{24}\left(f_{i, j-1}+22 f_{i, j}+f_{i, j+1}\right) .
\end{aligned}
$$

The bowtie-shaped stencil depicted in Fig. 1 is employed to conduct the high-order numerical evaluation of the surface integrals in (4). For the left half of the finite-volume cell, $S_{1}$,

$$
\iint_{S_{1}} E_{z}(x, y) d x d y=\sum_{i, j} C_{1 i, j} E_{z i, j} \Delta x \Delta y+O\left((\Delta x)^{7}+(\Delta y)^{7}\right)
$$

where $C_{1 i, j}$ is the weighting coefficient associated with each stencil point in the left of the planar interface. The integral for the right half of the cell, $S_{2}$, can be approximated in the same manner. Therefore, applications of these high-order spatial approximations, combined with the fourth-order staggered backward differentiation method for temporal integration in (4), result in the semiimplicit update equation for the interface electric field component, given as

$$
\begin{aligned}
\widehat{E}_{z i_{0}, j_{0}}^{n+1}= & -\frac{\varepsilon_{1}}{K_{i_{0}, j_{0}}} \sum_{i, j \neq i_{0}, j_{0}} C_{1 i, j} E_{z i, j}^{n+1}-\frac{\varepsilon_{2}}{K_{i_{0}, j_{0}}} \sum_{i, j \neq i_{0}, j_{0}} C_{2 i, j} E_{z i, j}^{n+1} \\
& +\frac{17}{22 K_{i_{0}, j_{0}}}\left[\varepsilon_{1} \sum_{i, j} C_{1 i, j} E_{z i, j}^{n}+\varepsilon_{2} \sum_{i, j} C_{2 i, j} E_{z i, j}^{n}\right] \\
& +\frac{9}{22 K_{i_{0}, j_{0}}}\left[\varepsilon_{1} \sum_{i, j} C_{1 i, j} E_{z i, j}^{n-1}+\varepsilon_{2} \sum_{i, j} C_{2 i, j} E_{z i, j}^{n-1}\right] \\
& -\frac{5}{22 K_{i_{0}, j_{0}}}\left[\varepsilon_{1} \sum_{i, j} C_{1 i, j} E_{z i, j}^{n-2}+\varepsilon_{2} \sum_{i, j} C_{2 i, j} E_{z i, j}^{n-2}\right] \\
& +\frac{1}{22 K_{i_{0}, j_{0}}}\left[\varepsilon_{1} \sum_{i, j} C_{1 i, j} E_{z i, j}^{n-3}+\varepsilon_{2} \sum_{i, j} C_{2 i, j} E_{z i, j}^{n-3}\right] \\
& +\frac{12 \Delta t}{22 \Delta y K_{i_{0}, j_{0}}}\left(\Psi_{x} H_{x_{0}, j_{0}-1 / 2}^{n+1 / 2}-\Psi_{x} H_{x i_{0}, j_{0}+1 / 2}^{n+1 / 2}\right) \\
& -\frac{12 \Delta t}{22 \Delta x K_{i_{0}, j_{0}}}\left(\Psi_{y} H_{x_{i_{0}}-1 / 2, j_{0}}^{n+1 / 2}-\Psi_{y} H_{x_{i_{0}+1 / 2, j_{0}}}^{n+1 / 2}\right) \\
& +O\left((\Delta x)^{4}+(\Delta y)^{4}+(\Delta t)^{4}\right)
\end{aligned}
$$


where

$$
K_{i_{0}, j_{0}}=\varepsilon_{1} C_{1 i_{0}, j_{0}}+\varepsilon_{2} C_{2 i_{0}, j_{0}} .
$$

In the fourth-order FDTD simulations employing this update scheme, late-time instability [11] is often observed to develop along the interface. To eliminate this numerical artifact without loss of accuracy, we apply a numerical stabilizer step, in which every electric field component, $\widehat{E}_{i_{0}, j_{0}}^{n+1}$ obtained by (8) at the interface is recalculated by another semi-implicit update equation. Therefore, the stable update for interface electric field components is achieved in two-steps. In the second step, a rectangular stencil, as depicted in Fig. 2 is employed instead to perform high-order numerical evaluation of surface integrals based on 2-D Lagrange interpolation. This stabilizer step can be written as

$$
\begin{aligned}
& E_{z i_{0}, j_{0}}^{n+1}=-\frac{\varepsilon_{1}}{L_{i_{0}, j_{0}}}\left[\sum_{i \neq i_{0}, j} D_{1 i, j} E_{z}^{n+1}{ }_{i, j}+\sum_{j \neq j_{0}} D_{1 i_{0}, j} \widehat{E}_{z}^{n+1} i_{0}, j\right] \\
& -\frac{\varepsilon_{2}}{L_{i_{0}, j_{0}}}\left[\sum_{i \neq i_{0}, j} D_{2 i, j} E_{z}{ }^{n+1}{ }_{i, j}+\sum_{j \neq j_{0}} D_{2 i_{0}, j} \widehat{E}_{z}^{n+1}{ }_{i_{0}, j}\right] \\
& +\frac{17}{22 L_{i_{0}, j_{0}}}\left[\varepsilon_{1} \sum_{i, j} D_{1 i, j} E_{z i, j}^{n}+\varepsilon_{2} \sum_{i, j} D_{2 i, j} E_{z i, j}^{n}\right] \\
& +\frac{9}{22 L_{i_{0}, j_{0}}}\left[\varepsilon_{1} \sum_{i, j} D_{1, j} E_{z i, j}^{n-1}+\varepsilon_{2} \sum_{i, j} D_{2 i, j} E_{z i, j}^{n-1}\right] \\
& -\frac{5}{22 L_{i_{0}, j_{0}}}\left[\varepsilon_{1} \sum_{i, j} D_{1 i, j} E_{z i, j}^{n-2}+\varepsilon_{2} \sum_{i, j} D_{2 i, j} E_{z i, j}^{n-2}\right] \\
& +\frac{1}{22 L_{i_{0}, j_{0}}}\left[\varepsilon_{1} \sum_{i, j} D_{1 i, j} E_{z i, j}^{n-3}+\varepsilon_{2} \sum_{i, j} D_{2 i, j} E_{z i, j}^{n-3}\right] \\
& +\frac{12 \Delta t}{22 \Delta y K_{i_{0}, j_{0}}}\left(\Psi_{x} H_{x_{0}, j_{0}-1 / 2}^{n+1 / 2}-\Psi_{x} H_{x_{i_{0}}, j_{0}+1 / 2}^{n+1 / 2}\right) \\
& -\frac{12 \Delta t}{22 \Delta x K_{i_{0}, j_{0}}}\left(\Psi_{y} H_{x i_{0}-1 / 2, j_{0}}^{n+1 / 2}-\Psi_{y} H_{x i_{0}+1 / 2, j_{0}}^{n+1 / 2}\right) \\
& +O\left((\Delta x)^{4}+(\Delta y)^{4}+(\Delta t)^{4}\right)
\end{aligned}
$$

where

$$
L_{i_{0}, j_{0}}=\varepsilon_{1} D_{1_{i_{0}, j_{0}}}+\varepsilon_{2} D_{2_{i_{0}, j_{0}}} .
$$

Note that on the right hand side of (10), nearby electric field values along the interface, $\widehat{E}_{i_{0}, j \neq j_{0}}^{n+1}$, predicted based on the bowtie-shaped stencil in the first step are used with weighting coefficients, $D_{1 i, j}, D_{2 i, j}$, for the stabilizer step.

For an interface electric field node near a perfect electric conductor (PEC) boundary, as illustrated in Fig. 3 , we can make a one-step update instead, based on the rectangular stencil, as shown, to compute the electric field value at $n+1$ time level. We can take advantage of the fact that the tangential electric field values at the PEC

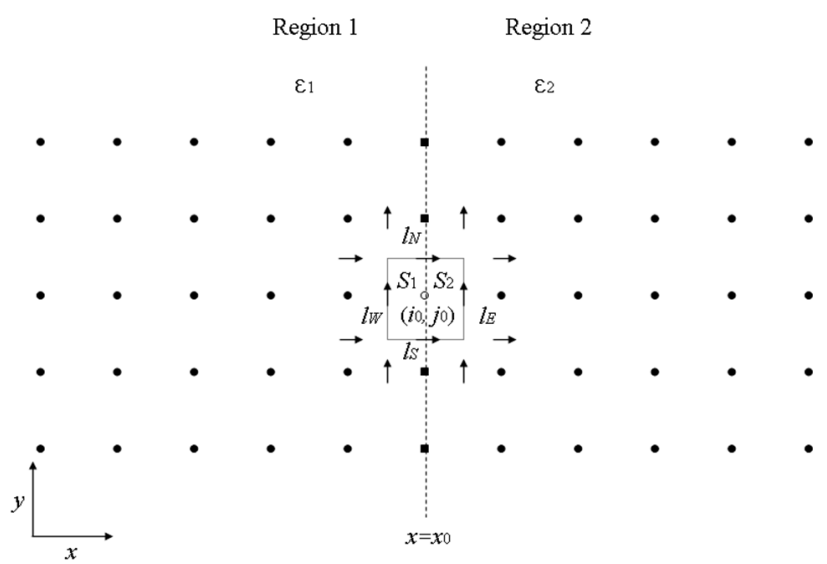

Fig. 2. Numerical stencil for the electric field, $E_{z_{i_{0}}, j_{0}}^{n+1}$, denoted by the unfilled circle at the interface in the stabilizer step. Filled circles indicate electric field nodes with known $n+1$ time level information and nearby filled squares along the interface represent electric field nodes with their $n+1$ time level electric field values predicted using the bow tie-shaped stencil.

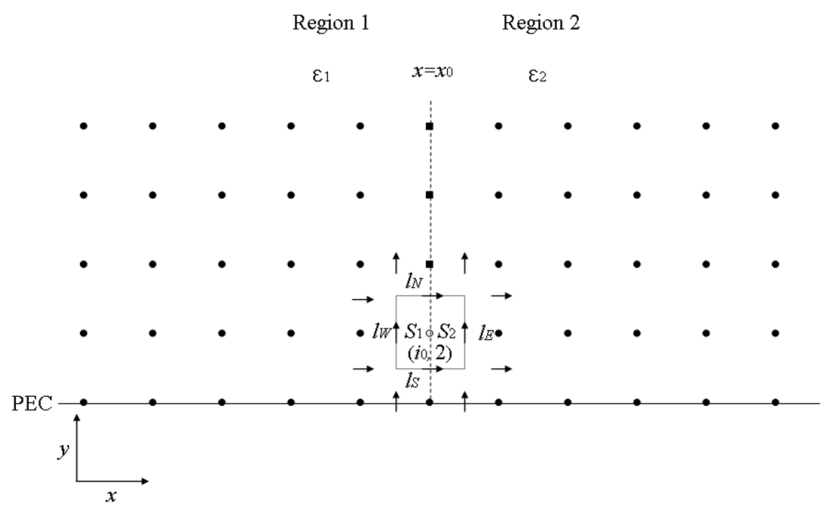

Fig. 3. Numerical stencil for the electric field, $E_{z i_{0}, 2}^{n+1}$, denoted by the unfilled circle at the planar dielectric interface near a PEC boundary. Filled squares along the interface represent electric field nodes with their $n+1$ time level electric field values predicted using the bowtie-shaped stencil.

boundary are always known to be zero and that nearby interface electric nodes, as denoted by the filled squares, are readily available by update steps based on the bowtie-shaped stencil described in Fig. 1.

\section{Numerical Validation}

We now focus on numerical validation of the fourthorder accurate planar dielectric boundary scheme, in combination with the fourth-order FDTD method [8], as presented in the previous section. To this end, the partially-filled cavity considered for the 2-D TE formulation 


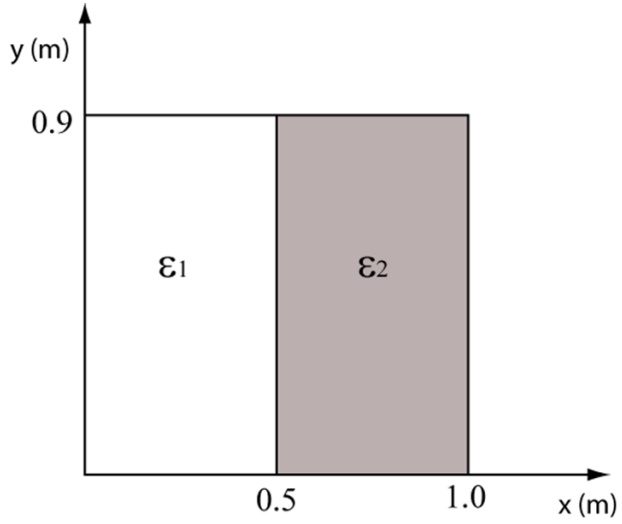

Fig. 4. Geometry of a 2-D partially-filled cavity with perfectly conducting walls. The right half of the cavity is filled with a lossless dielectric material with the relative permittivity, $\varepsilon_{r}=4$.

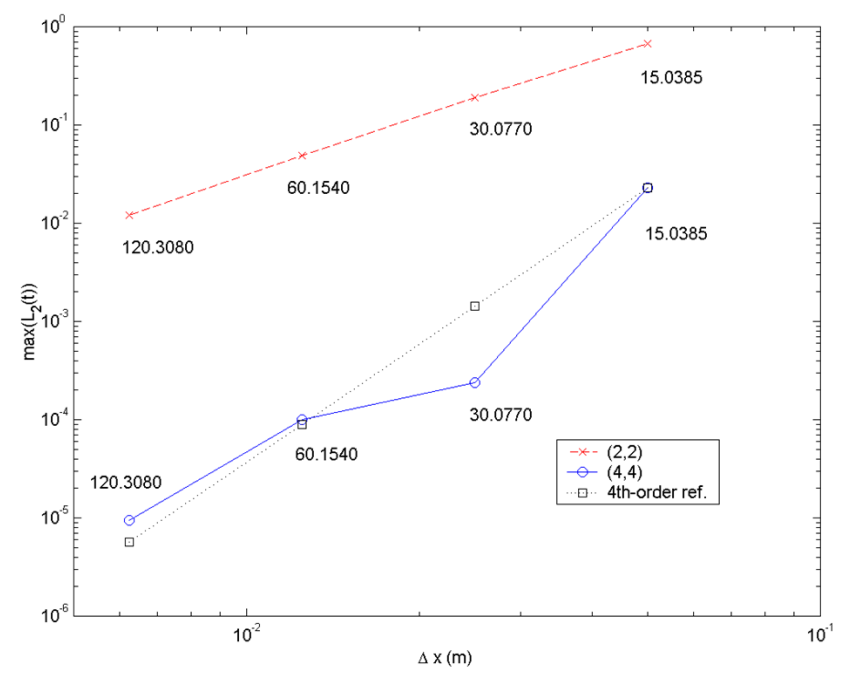

Fig. 5. Convergence slopes for the 2-D inhomogeneous cavity.

[8] is revisited to conduct a convergence test of the proposed scheme in the 2-D TM case, as depicted in Fig. 4. The $\mathrm{TE}_{3,2}$ mode is imposed as an initial condition and the $L_{2}$ norms of the global errors in the computed electric field, $E_{z}$ are monitored for 20 periods of the resonant frequency, $f_{r}$, which is found to be $398.79 \mathrm{MHz}$ for the mode under study. The convergence rates and computational costs of Yee's second-order, or $(2,2)$ scheme and the proposed fourth-order, or $(4,4)$ scheme are carefully compared, as shown in Fig. 5 and Fig. 6, respectively. For all numerical tests in this paper, the time step for the second-order scheme, $\Delta t_{(2,2)}=0.95 \Delta t_{Y e e}$ and the time step for the fourth-order scheme, $\Delta t_{(4,4)}=0.4 \Delta t_{Y e e}$, where $\Delta t_{Y e e}$ is the maximum time step for Yee's second-order FDTD method derived from the stability analysis [1]. The numbers near the data points in these plots indicate grid points per wavelength in free space.

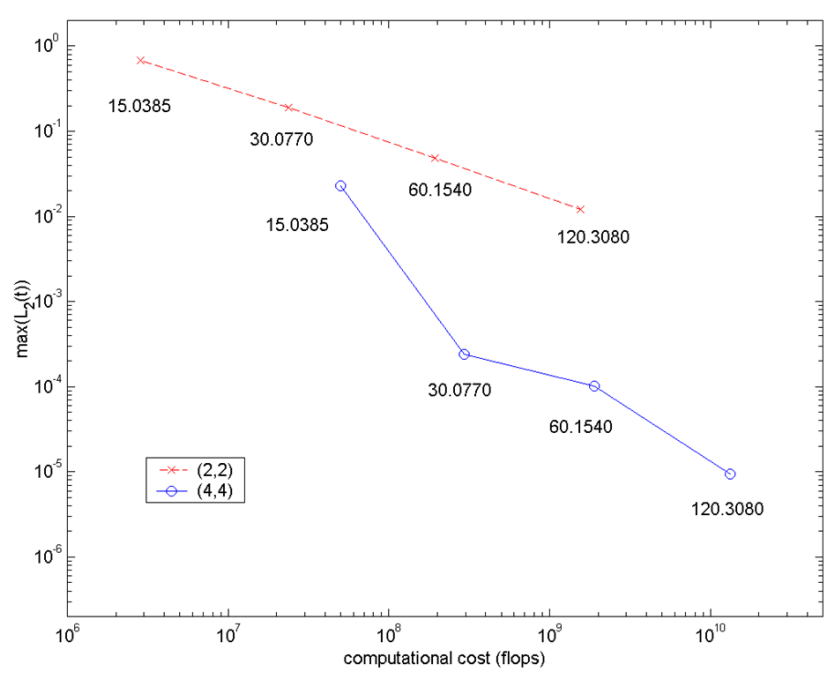

Fig. 6. Computational costs for the 2-D inhomogeneous cavity.

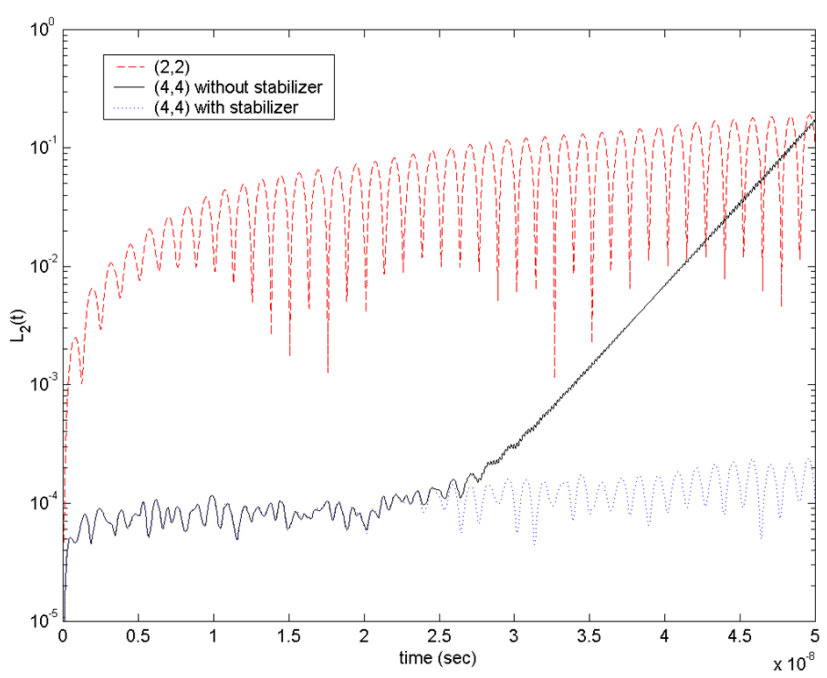

Fig. 7. Temporal history of the $L_{2}$ norm errors.

Thus, in the dielectric region $\left(\varepsilon_{r}=4\right)$, the number of grid points per wavelength is reduced by half. It is also assumed that $\Delta x=\Delta y$ in all simulation runs.

Fourth-order convergence is clearly observed in Fig. 5 for the fourth-order scheme with the proposed interface scheme. The superb accuracy of the proposed $(4,4)$ scheme is demonstrated in this comparison with the conventional $(2,2)$ scheme. For example, the $(4,4)$ scheme with the grid resolution of about 15 points per wavelength in the dielectric outperforms the $(2,2)$ scheme by more than two orders of magnitude in terms of the global numerical error. This translates to a huge computational cost difference, as noted in Fig. 6. The benefit of the stabilizer step in the proposed fourth-order boundary scheme is evident in Fig. 7, which shows the temporal history of numerical errors from three different schemes. In this example, $\Delta x=\Delta y=0.025 \mathrm{~m}$, which corresponds to 
about 15 grid points per wavelength in the dielectric for the given resonant frequency. The $(4,4)$ scheme with the stabilizer shows superior accuracy compared to the $(2,2)$ scheme, while the $(4,4)$ scheme without the stabilizer exhibits an exponential growth of the non-physical solution after about 10 periods. Therefore, application of the stabilizer technique in the interface update is the key to sustain stability of the $(4,4)$ scheme without loss of accuracy. Long-time stability of the $(4,4)$ scheme with the proposed interface scheme has been numerically verified by running the implemented code for the 2-D partially-filled cavity for one million time steps with various discretizations and no late time instability has been found in the computed solutions.

\section{Conclusions}

A numerical boundary scheme for the planar dielectric interface has been presented to extend the previously proposed fourth-order FDTD method to the 2-D TM case. Numerical results from a 2-D inhomogeneous cavity have shown excellent computational efficiency of the proposed fourth-order method as well as its promising potential for highly accurate full-wave time domain simulations of electrically large structures. Efforts are underway to extend capabilities of the proposed fourth-order FDTD algorithm to deal with more complex geometries.

\section{References}

[1] A. Taflove, Computational Electrodynamics: The Finite Difference Time-Domain Method, Artech House, Norwood, MA, 1995.

[2] A. Taflove, Advances in Computational Electrodynamics: The Finite Difference Time-Domain Method, Artech House, Norwood, MA, 1998.

\section{Kyu-Pyung Hwang}

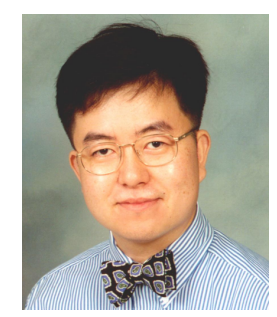

received the B.S. degree in electronic engineering from Seoul National University, Seoul, Korea, in 1993, the M.S. degree in electrical engineering from the Ohio State University, Columbus, OH, in 1996, and the Ph.D. degree in electrical engineering from the University of Illinois at UrbanaChampaign, Urbana, IL, in 2002. Upon his graduation, he joined Intel Corporation in Chandler, Arizona, USA. He has been with Samsung Electro-Mechanics Co., Ltd in Suwon, Korea since 2007. His research interests are in computational electromagnetics, numerical partial differential equations, and advanced microelectronic packaging.
[3] A. Yefet, P. G. Petropoulos, "A staggered fourth-order accurate explicit finite difference scheme for the time-domain Maxwell's equations," J. Comput. Phys., vol. 168 , no. 2, pp. 286-315, Apr. 2001.

[4] Z. Q. Xie, C. H. Chan, and B. Zhang, "An explicit fourth-order staggered finite-difference time-domain method for Maxwell's equations," J. Comput. Appl. Math., vol. 147, pp. 75-98, 2002.

[5] Z. Q. Xie, C. H. Chan, and B. Zhang, "An explicit fourth-order orthogonal curvilinear staggered-grid FDTD method for Maxwell's equations," J. Comput. Phys., vol. 175, pp. 739-763, 2002.

[6] J. S. Hesthaven, "High-order accurate methods in time-domain computational electromagnetics: A review," Adv. Imag. Electron Phys., vol. 127, pp. 59123, 2003.

[7] S. Zhao, G. W. Wei, "High-order FDTD methods via derivative matching for Maxwell's equations with material interfaces," J. Comput. Phys., vol. 200, pp. 60103, 2004.

[8] K.-P. Hwang, "A fourth-order accurate FDTD scheme with long-time stability," IEEE Microw. and Wireless Compon. Lett., vol. 15, no. 4, pp. 271-273, Apr. 2005.

[9] K.-P. Hwang, J.-Y. Ihm, "A stable fourth-order FDTD method for modeling electrically long dielectric waveguides," J. Lightw. Technol., vol. 24, no. 2, pp. 10481056, Feb. 2006.

[10] M. Ghrist, B. Fornberg, and T. Driscoll, "Staggered time integrators for wave equations," SIAM J. Numer. Anal., vol. 38, pp. 718-741, 2000.

[11] C.-T. Hwang, R.-B. Wu, "Treating late-time instability of hybrid finite-element/finite-difference timedomain method," IEEE Trans. Antennas Propagat., vol. 47, no. 2, pp. 227-232, Feb. 1999. 